\title{
Aprender a leer y escribir en Chihuahua: siglo XIX
}

\author{
Francisco Alberto Pérez Piñón \\ Guillermo Hernández Orozco \\ Jesús Adolfo Trujillo Holguín
}

Profesores investigadores Universidad Autónoma de Chihuahua

\section{Resumen}

I objetivo del presente trabajo es $\checkmark$ mostrar, desde esta región, otras 1 interpretaciones apoyadas en documentos históricos que rompen con las generalizaciones que hacen parecer que los procesos para la enseñanza de la lectura y la escritura en el siglo XIX se realizaron de manera unificada en nuestro país, perdiéndose la riqueza y la difusión de los esfuerzos realizados desde estos apartados lugares, por lo que es apasionante hacer un viaje al pasado que nos ilustre en el conocimiento de las formas que se idearon en el siglo XIX para aprender a leer y escribir, así como rescatar los textos que se utilizaron en la época; los catones, cartillas y el método educativo con el que se trabajaba, así como el catecismo. En Chihuahua, México, tuvimos en la primera parte del siglo XIX, grandes personalidades francesas (Guignor, Roussy) que tuvieron gran impacto en la educación, obteniendo con ello las ideas de avanzada en lo educativo e innovando en la lectura y la escritura; como el caso de Laubscher, de nacionalidad alemana, con la introducción de la enseñanza intuitiva en el Estado y las Conferencias Pedagógicas para la formación de profesores antes de la creación de la escuela normal y su método de lectura y escritura de corte fononímico; así como la presencia en estas tierras norteñas de maestros discípulos de Enrique C. Rébsamen.

Palabras clave: educación, lectura, escritura, cartillas, catones. 
REVISTA DE INVESTIGACIÓN EDUCATIVA DE LA REDIECH N. 9 ISSN: 2007-4336

\section{Introducción}

En estos tiempos de posmodernidad, marcados por distintos teóricos en el campo de la disciplina histórica, en el cual se está dando el viraje de los estudios como narración de los hechos del pasado y su acercamiento a ellos mediante cuidadosas epistemologías para conocer los fenómenos y su reconstrucción hacia una historia más inmediata, una historia del tiempo presente y que migre de lo epistémico a lo ontológico para dar cuenta de los sujetos y sus prácticas cotidianas en la construcción de la sociedad, no podemos desprendernos aún de rescatar la memoria colectiva del pasado, memoria que interpretamos y tratamos de acercarnos a lo más verosímil en su reconstrucción, sin pensar que esa es la verdad unívoca y sí en un pensamiento abierto de que será otra más de las interpretaciones que se hayan presentado o que se harán en un futuro; por varias razones, entre ellas, rescatar a través de sus huellas los acontecimientos del pasado y tenerlos presente para conocer el desarrollo en relación a la escritura y lectura en nuestro estado de Chihuahua.

Con la mirada puesta en el posmodernismo, que clarifica que el pasado no existe como entidad física, sino como interpretaciones, queremos abonar con una nueva perspectiva, que dé cuenta de nuestra historia regional concreta, a la manera de las microhistorias y en respuesta a las macrohistorias que hemos internalizado como ideas generalizadoras que ocultan lo singular y ocurrido de forma sui géneris.

Con el presente ensayo se pretende rescatar nuestra memoria histórica partiendo de lo local, pero sin menoscabo de su integración en el contexto nacional e internacional, por la razón de que las ideas en educación no son exclusivas de una sola región o país, sino que son ideas que han viajado y continúan viajando entre el viejo y el nuevo mundo a través de distintas mediaciones que dan cuenta de ellas; las evidencias que permiten la elaboración del presente escrito son de fuentes primarias encontrados en los archivos, así como de fuentes secundarias que están relacionadas con las formas de enseñar la lectura y la escritura en el siglo XIX, a través de las cartillas y los catones con el método del catecismo, para hacer la analogía con lo acontecido en nuestro contexto.

El acercamiento a las fuentes se realizó de manera lógica, para lo cual fue necesario efectuar la búsqueda de los documentos que sustentan la narrativa histórica, lo que permitió ubicar el objeto de trabajo, para pasar a hacer una concatenación de las evidencias y así dar cuenta de la reconstrucción de los acontecimientos y posteriormente proceder a sus valoraciones, con el fin de pasar de la simple descripción a la comprensión y transformación del objeto histórico, mediante nuevas interpretaciones.

\section{Desarrollo}

La colonización en el siglo XV y la posterior conquista por parte de los españoles de los territorios de lo que se conocería como la Nueva España, hicieron necesaria la creación de una cultura que justificara la existencia de culturas superiores, en detrimento de las autóctonas, con fines de subyugación; fue por ello apremiante la incorporación de la población indígena a la lectura y la escri- 
tura, e hizo necesario "desde los comienzos de la colonización, el envío de cartillas desde la península española al nuevo mundo, se hizo en grandes cantidades con destino a la enseñanza de los hijos de españoles y de los naturales. Junto con las cartillas llegaron también remesas de catones para los párvulos" (Torre, 1960: 214) Las cartillas podemos considerarlas el primer instrumento formal para la enseñanza de la lectura y posteriormente la escritura, era un cuaderno pequeño que contenía las letras del alfabeto y frases cortas con el fin de que se realizara su lectura, el número de páginas iban desde las ocho como en el caso de la "Cartilla y arte brette y bien copendioso para enseñar a deletrear y leer perfectamente y con mucha facilidad y con todas o las más abreviaturas que se supieron hallar" (231), hasta 32 hojas como en el caso de la "cartilla para enseñar a leer a niños con la doctrina christiana que se canta, Amados hermanos./ Agora de nuevo examinada, corregida y enmendada: y con privilegio de su/ Magestad" (233), cartilla llegada a América en el año de 1606, la cual contenía el alfabeto y sonidos para las vocales, las oraciones al señor, el ave maría, credo y el salve regina.

Los enunciados de estas dos cartillas nos permiten hacer la interpretación del método o la forma que se utilizaba para enseñar a leer, la cual era el deletreo, ya que deberían de aprenderse las letras por su nombre.

La fuerza de las frases y lecturas de las cartillas hacían alusión a la deidad y los santos, planteándose la transmisión de la doctrina cristiana como campo de conocimientos, la forma de enseñanza del dele- treo estaba basada en los catecismos, entre dichos textos, los más conocidos y citados en el siglo XIX en Chihuahua fueron el del padre Ripalda y el del Abate Fleury, con el fin de transmitir las enseñanzas de la religión católica; un ejemplo del catecismo del padre Ripalda es el siguiente:

¿Sois Christiano?

Sí por la gracia de nuestro señor Jesucristo

¿Qué quiere decir Christiano?

Hombre que tiene la fe de Christo, que profesó en el bautismo.

También existían los catecismos laicos como el de economía política que aún conservamos en el archivo histórico de nuestra universidad, a manera de ejemplo de su enseñanza era en forma de preguntas y respuestas.

¿Qué nos enseña la economía política?

Nos enseña cómo se producen, se distribuyen y consumen las riquezas en la sociedad.

¿Qué se entiende por esta palabra "riquezas"?

Puede entenderse la significación de esto como...

"La enseñanza de la lecto-escritura hasta unos años se había practicado básicamente con los viejos sistemas de deletreo o silabeo, el primero había predominado hasta el siglo XVIII y el segundo se utilizó en los siglos XIX y XX, aunque en muchos años se usaron indistintamente" (Aguirre y Rodríguez, 2008: 54).

Queda claro que uno de los primeros métodos para la enseñanza de la lecto-escritu- 
REVISTA DE INVESTIGACIÓN EDUCATIVA DE LA REDIECH N. 9 ISSN: 2007-4336

ra fue el deletreo, no se requiere de muchos rodeos interpretativos para asentar que lo primero que se debía hacer para aprender a leer era conocer las letras del abecedario, como punto de partida para iniciarse en el simbolismo.

Barbosa (1998) nos menciona, que no bastaba conocer las letras, sino que también había que memorizarlas, solo que existía un gran problema, se presentaban dificultades cuando las letras se deletreaban por su nombre: a, be, ce, che, de, e, efe, ge, ache, etc. y posteriormente se trataba de leer en palabras, frases y enunciados ya como estructuras, un ejemplo que el mismo autor atribuye a Enrique Rébsamen, ya pasada la segunda mitad del siglo XIX cuestionaba el método de deletreo con el siguiente ejemplo: ache - i - jota - o, solo podía leerse como acheijotao, cuando en realidad la palabra es hijo con el cual de manera natural los niños estaban en constante interacción.

En los años de 1824 a 1831 las escuelas que funcionaban tan solo en la capital (Chihuahua), eran según la obra de Hernández (1999):

La escuela principal o también llamada lancasteriana, la escuela subalterna y la particular titulada por sus creadores casa de las cátedras de Cipriano Irigoyen y su ayudante Jorge García, así como la de Guillermo Roussy, de Víctor Sáenz y Pedro de Lille y Felipe López; estas 7 de primeras letras para niños y para niñas hubo 2, la escuela pública para niñas y a partir de 1831 la particular de la maestra Betancourt, sin contar las escuelas que funcionaban con reducido y variado número de estudiantes, llamadas de la amiga, entre las que se encontraban las de las maestras María Casmira Castillo; María Guadalupe Terrazas; Faustina Castro; María Soledad Castro; Marcela Grielo y Martha Bastarda del Pozo (19).

Es el sistema lancasteriano el que se implanta en el país, método revolucionario en su tiempo, representaba toda una serie de cambios, permitía:

...simultáneamente lectura, escritura y aritmética. Los grupos numerosos se dividían en clases de acuerdo con el grado de conocimientos adquiridos, que se ponían a cargo de los niños más adelantados, todo en un aula grande bajo la supervisión del preceptor. El método se basaba en un complejo conjunto de normas, ritos escolares y tareas, así como en un sistema de premios y castigos" (Arredondo, 2003: 107).

El sistema lancasteriano fue traído a Chihuahua, por Antonio Cipriano Irigoyen encargado de la escuela de niños, cuando acudió a la ciudad de México en el año de 1824 y trae la visión de crear una escuela normal que encarara el reto de formar preceptores. El presbítero Cipriano Irigoyen en el año de 1825 escribe con puño y letra el instructivo al que se debían suscribir las escuelas públicas de Chihuahua. La escuela normal no se creó como la percibimos hoy en día, de conformidad con el sistema lancasteriano se fortaleció el segundo departamento dedicado a la formación de preceptores; el primer departamento estaba enfocado a la enseñanza de las primeras letras y el tercer departamento como se afirma en Pérez (2007: 21) "se proporcionaba la enseñanza y la instrucción de artes y oficios y 
se preparaba para la educación superior al proporcionar las asignaturas de latinidad, dibujo lineal, matemáticas, historia, geografía y francés". Estos tres departamentos funcionaron en la escuela principal creada en el año de 1825.

Por estas norteñas tierras nos llegó la influencia del lancasterianismo en su versión más liberal, de conformidad con la adaptación que realizó el padre Irigoyen en la ciudad de Chihuahua, los procesos de la lectoescritura estaban centrados en "la lectura se aprendía por deletreo, los de la primera clase aprendían las letras, la segunda clase las sílabas, de dos a cinco sílabas posteriormente se pasaba al vocabulario de palabras enteras y la lectura corrida en libros" (Hernández, 1999: 9).

Se inauguraba una nueva época en la forma de enseñar la lectura y la escritura a la gran masa de analfabetas, aunque se sigue manejando el sistema del conocimiento de las letras de forma sintética y después se procede al análisis al juntarlas en sílabas y después palabras y frases, se estaba pasando ya al silabeo, conforme se menciona en el informe del preceptor Antonio Cipriano Irigoyen en el cual se destaca que tenía en la escuela principal 48 en la primera clase (deletreando) de segunda clase 28 leyendo cartilla, 40 en catón, 81 escribiendo en papel, 17 escriben y 40 de silabario.

El silabeo estaba tomando auge y las cartillas más conocidas para este tipo de lecto-escritura, fueron las del silabario de San Miguel según lo menciona Barbosa (1998). Dicho silabario contenía ocho páginas, no omitimos lo rudo del método lancasteriano que consistía en la memorización y en los castigos que se realizaban cuando alguien no aprendía las lecciones encomendadas por el monitor en el tiempo establecido, un ejemplo de ello es que etiquetaba en tarjetas la falta cometida y el castigo a cumplir colgándoselos del cuello o la clásica arrodillada poniendo los brazos en forma de cruz y sosteniendo sin bajarlos objetos pesados en ambas manos a criterio del preceptor; o las burlas de poner a los estudiantes más atrasados las orejas de burros, como formas de castigo; sin embargo, en Chihuahua en el capítulo 11 del Reglamento escrito por Cipriano Irigoyen ya abolía los castigos, aunque no por ello podemos decir que ya no ocurrían, pero al menos es una forma liberal que se apartaba del lancasterianismo clásico.

Esta forma de enseñanza del silabeo partía de conocer primero las vocales. Posteriormente se realizaban combinaciones con las consonantes, de menor a mayor dificultad (B F M P) BA FA MA PA y así se seguía la conjugación con cada una de las vocales. Se realizaba un juego de relaciones de sílabas para formar palabras y posteriormente la relación entre palabras.

Aunque el método expresado en el Silabario de San Miguel fue utilizado en escuelas parroquiales y religiosas también se utilizó en las escuelas auspiciadas por el gobierno y particulares. El silabario tiene un gran avance en relación al deletreo, puesto que permite un acercamiento fonético en las sílabas y las sumas de éstas con las palabras y estructuras más amplias. Debemos mencionar que el deletreo no siempre era malo, cuando éste se hacía en relación al so- 
nido de las letras y que el silabeo se demeritaba cuando se pasaba al nombre de las letras en las sílabas.

Se gestaban cambios en la escuela de Chihuahua; la escuela principal había tenido por directores a Antonio Cipriano Irigoyen; Faustino Irigoyen; José María Sánchez, Félix Madariaga; Víctor Sáenz quien en el año de 1832 deja la dirección y ésta es ocupada por el francés Bernardo Guignor, contratado por el erario público para que reestructure la escuela principal a la cual cambió el nombre por el de Escuela Normal, y le dio un cambio en la formación de preceptores, ya que éstos a partir de las directrices de Guignor se formarían por la observación práctica, y por supuesto por las enseñanzas que trabajaba con ellos; estas enseñanzas partían de la ilustración francesa y de los métodos objetivos que se estaban poniendo en práctica en Europa, métodos que consideraban que la educación tenía que ser acorde a la naturaleza y el contexto de los niños. Guignor realiza una gran obra educativa en Chihuahua, al reestructurar y reforzar la formación de maestros, se imponen nuevas ideas y directrices a la educación en Chihuahua, un gran avance, se promueve al tomar en cuenta los intereses y la naturaleza de los niños para el acceso a las primeras letras, se empiezan a dar las contradicciones de lo duro de las enseñanzas basadas en el método lancasteriano y ahora con ideas europeas de la libertad, fraternidad e igualdad traducidas a lo educativo.

A la salida de Guignor ocupa la dirección de la escuela principal, otro francés, Juan José Capoulade quien llega a Chihuahua con su esposa Luisa Maulía quien se ocupa- rá de la dirección de la escuela de niñas, la influencia de las ideas de estos franceses en la educación en Chihuahua permitirán el arribo a una sociedad a la que aspiraban los grupos liberales; pero la coronación de estas ideas serán con Guillermo Roussy otro francés que arriba a estas tierras en la primera mitad del siglo XIX; había realizado en el año de 1837 en la ciudad de México un trabajo de gran calidad, resumía su método de enseñanza impreso en la guía Roussy (1837) de las escuelas primarias en la parte primera, en la cual explicita lo concerniente a la lectura y la escritura, se destaca:

En esta primera parte allano una dificultad en la lectura, dando a las letras el nombre del sonido que tienen en la horación. Doi el abecedario reducido según la ortología moderna, i entresaco los vicios introducidos en la pronunciación. Reduzco el deletrear a sus verdaderos límites arreglo las lecciones a la naturaleza de la lectura, la ligereza de los niños ni su escasez de ideas, prolongo los ejercicios, a fin de que los niños no los aprendan de memoria y tengan que cautivar siempre la atención; los conbino de manera que hablan al oído i a la vista, conportando y comparando los signos que tienen relación alguna, ya en la figura, ya en el sonido, de manera que los alumnos se puedan ejercitar por si solos según el fin del método; proporciono al maestro el instrumento, le dejo nomás el cuidado de hacer trabajar.

Para reducir la problemática de la lectura, debe de ser centrada en el fonetismo, el sonido que las letras obtienen al conjugarse con la suma de otras, se adecuaban las lecturas a los referentes con los cuales estaba en contacto el niño, de manera que éstas le 
hablaran al percibirlas por el oído y la vista, en la práctica estamos hablando de un método natural el cual requiere de la observación y manipulación de los objetos para su reconocimiento y posteriormente hacer su representación simbólica, esto para el caso de la enseñanza de una educación que partiera de la realidad de los estudiantes.

La presencia y las ideas innovadoras de los franceses educadores Guignor, $\mathrm{Ca}$ poulade, Maulía, Roussy gestaron una corriente de ideas frescas, modernas y sobre todo útiles en la formación de la sociedad chihuahuense a la vez que prepararon el camino para que estas ideas se continuaran ahora con la llegada de nuevos educadores europeos como es el caso de Enrique Laubscher quien "por encargo del gobierno de Chihuahua realiza un diagnóstico de la educación" (Pérez, 2007: 27) y establece las Conferencias Pedagógicas, mismas que dictó en Chihuahua a los entonces profesores encargados de las escuelas de primeras letras, centradas en la enseñanza objetiva y en el fonetismo como método de lectura y escritura. Laubscher había publicado en 1880 su manual práctico para la enseñanza objetiva, utilizando por primera vez el método simultáneo como complemento de la enseñanza objetiva.

En 1892, llega a Chihuahua Alberto Vicarte un discípulo de Rébsamen y difundirá el método fonético entre los profesores chihuahuenses. La obra educativa chihuahuense se consolida con las visiones educativas modernas alimentadas en el siglo XIX con la visión francesa; con la visita y las conferencias pedagógicas del alemán naturalizado mexicano Enrique Laubscher y la oleada de profesores formados en la normal para profesores de la ciudad de México y los alumnos de Rébsamen egresados de la escuela normal de Jalapa, podemos considerarnos herederos de una educación moderna que se estaba poniendo en práctica a nivel mundial.

Con los aportes de Laubscher y de Rébsamen en relación al método fonético o Rébsamen como se le ha llegado a conocer, es Vicarte quien nos traduce el cambio en el proceso de la lectura y la escritura; dicho método descansa en tres principios: la simultaneidad para el abordaje de la lectura y escritura de marcha analítico sintética ya que se parte de estructuras de palabras familiares al niño, para su descomposición en sílabas, letras (asociadas fonéticamente al contenido de las palabras), palabras y después proceder a su composición estructural.

En el año de 1906 (Aguirre y Rodríguez, 2008) surge el método del Profesor Gregorio Torres Quintero denominado Onomatopéyico que partió como su autor lo menciona de una adaptación del Orbis Pictus obra de Comenio para la enseñanza de las lenguas, método que regresa a las marchas sintético analíticas para la enseñanza de la lectura y la escritura; la polémica fuerte que se suscita en la época es en relación a los procesos de la marcha sintética analítica cuando en el siglo XIX se había tomado como universal el procedimiento de la marcha analítico sintética .

Un ejemplo para ilustrar el método onomatopéyico y que difícilmente podemos olvidar, partía de los sonidos que producían 
las cosas generalmente de las más conocidas por el niño, mismas que se hacía su representación gráfica con ilustraciones $o$ directo con la naturaleza para representarlas simbólicamente, objeto nombre y posteriormente se partía del análisis fonético de las letras para llegar a la composición de estructuras más complejas.

Los métodos de deletreo; silábicos; fonológicos, como el Rébsamen y el onomatopéyico, permitieron la alfabetización de los chihuahuenses y difícilmente hoy en día los maestros podemos desprendernos en nuestra enseñanza de la lectura y la escritura de tan valiosas aportaciones.

\section{Conclusiones}

Se espera que el presente recorrido histórico que se realiza en el cuerpo del trabajo, como un esfuerzo por contribuir al rescate de las distintas formas que se instrumentaron para la enseñanza y aprendizaje de la lectura y escritura resulte ilustrativo y motive a la vez nuevas interpretaciones, que en parte y por la herencia colonizadora recibimos los pueblos latinoamericanos.

Es seguro que el rescate de las formas de lectura y escritura antes descritas no conlle- ven a la formación de lectores, sin embargo, como conclusión final podemos afirmar que un buen método de lectura y escritura asociado a una educación objetiva, práctica o significativa haciendo alusión a Ausubel, puede llevarnos a la formación de personas interesadas por la lectura, que tanta falta hacen en nuestra sociedad.

\section{Referencias}

Aguirre L. y Rodríguez, M. (2008). El método onomatopéyico, un diálogo a la distancia de Torres Quintero con Comenio. SOMEHIDE, Memoria, conocimiento y utopía. 5.

Arredondo A. (2003). Obedecer, servir y resistir. La educación de las mujeres en La historia de México. UPN, México: Editorial Miguel Ángel Porrúa.

Barbosa, A. (1998). Cómo han aprendido a leer y a escribir los mexicanos. México: Editorial Pax.

Hernández, G. (1999). El Instituto Científico y Literario de Chihuahua 1827-1850. Textos Universitarios. UACH.

Pérez, F. (2007). Contribución educacional de la escuela normal del estado de chihuahua en el período de la revolución mexicana. Textos universitarios. Universidad Autónoma de Chihuahua.

Roussy, G. (1837). Guía de las escuelas primarias parte primera. Méjico. Impreso por Miguel González. Calle de cadena número 13. Archivo histórico municipal fondo 1830-1840.

Torre, R. (1960). Thesaurus, Tomo XV, números 1,2 y 3. Versión electrónica recuperado en: http://cvc.cervantes.es/lengua/thesaurus/pdf/15/TH_15_123_222_0. pdf. 